\title{
Interprofessional Education: in silo, ineffective
}

\author{
Salomeh Salari $^{1}{ }^{1} \cdot$ Seth Klapman $^{1} \cdot$ Brian Fry $^{1} \cdot$ Stephanie Hamel $^{2}$
}

Published online: 19 September 2017

(C) International Association of Medical Science Educators 2017

\section{Introduction}

Optimal patient-centered care requires healthcare professionals to work as a cohesive team [1]. At its best, interprofessional teamwork (IPT) empowers each individual to contribute his or her expertise regardless of job title or degree and allows for seamless collaboration. In order to successfully incorporate IPT into the practice of patient care, interprofessional education (IPE) must be well integrated into undergraduate medical curricula [1]. IPE is already widely recognized as a vital component of medical education. However, institutions tend to incorporate IPE into their curriculum in silos, with single workshops or simulations rather than longitudinal experiences spanning across the 4 years of medical undergraduate training [1]. Our institution, the University of Michigan, is no exception. A new IPE course for first-year medical students, the Interprofessional Clinical Experience (ICE), allows students to shadow different healthcare professionals within an assigned department or clinic for a semester. While wellintentioned, this siloed, one-dimensional approach presents IPT as an isolated skill, rather than a habit of practice. Without active participation and repetitive exposure to the various roles in healthcare, students cannot fully comprehend the scope of IPT.

When students are thrust into the complex environment of the hospital in their clinical years, they are expected to

Salomeh Salari

ssalari@med.umich.edu

1 University of Michigan Medical School, 1135 Catherine Street, Ann Arbor, MI 48109, USA

2 Hospital of the University of Pennsylvania, 3400 Spruce Street, Philadelphia, PA 19104, USA navigate the boundaries of various healthcare members' roles in addition to adjusting to the responsibilities of patient care. Medical students can effectively hone their clinical skills only when they have been adequately trained to work with other healthcare workers such as nurses, social workers, pharmacists, and physician assistants. Below, we outline components of IPE that are missing in current medical school curricula, including longitudinal, active collaboration between various health professional schools and individual empowerment to speak up for more accurate and efficient patient care.

\section{Interprofessional Collaboration}

Medical curricula must include IPE as a fundamental part of both the preclinical and clinical years to establish a learning environment supportive of dynamic collaboration. Currently, medical undergraduate training is centered on individual growth and achievement. While it remains vital for medical students to hone the traditional skills of a practicing clinician (e.g. thorough history taking and physical exam maneuvers), the transition from individual to team-based patient care requires medical schools to place an emphasis on group problem solving. One way to address this would be overlapping or sharing curricula between health professional schools to provide students with the ability to practice effective IPT [2].

In the preclinical years, regularly scheduled didactic lectures, small group learning, clinical simulations, and directed shadowing experiences can expose medical, nursing, pharmacy, and social work students to the shared foundational material of their future professions in a team-based manner. Students can form collaborative relationships with their interdisciplinary classmates, which can be done while learning to function in various roles within assigned teams [3]. Examples of such exercises may include problem-based clinical skill development, exploration into the ethical and psychosocial 
elements of medical care, or guided simulation of patient care scenarios [2].

Segueing to the clinical years, students should have opportunities to participate in healthcare experiences such as interdisciplinary rounds, direct patient care interactions, and "huddle" style meetings with team members. In addition to learning how to function in the clinical environment and share responsibility for the care of specific patients, emphasis must be placed on interpersonal and team development. These activities will naturally introduce opportunities to evaluate healthcare processes and systems as a whole, leading to possible research, simulation, and professional development opportunities that will further enhance the preparation of future medical professionals. Incorporating IPE longitudinally allows for optimization of IPT by playing to individual strengths and streamlining the practice of medicine to the benefit of all patients.

In order to best instill IPT as a standard of care, it is important to first identify programs that have excelled in various aspects of IPE. Rosalind Franklin University has initiated a first year course for medical students that puts them in teams with individuals from allopathic and podiatric medicine, medical radiation physicists, nurse anesthetists, pathologists' assistants, psychologists, and physician assistants. If students wish, they can extend their learning through their clinical years [4]. The University of Washington engages students in interprofessional team simulations in which students from medicine, nursing, and pharmacy provide urgent care to simulated patients [4]. The University of Michigan is launching a clinical decision-making course allowing dental, pharmacy, social work, and medical students to meet weekly to discuss patient cases and work though clinical scenarios together. In order to better provide a framework for implementation of IPE, it is imperative to collaborate between institutions, gleaning what has worked, and more importantly, what has not worked from existing IPE curricula.

\section{Individual Empowerment}

Maximizing the benefits of IPT includes not only seamless collaboration within a team but also empowering individual providers to take initiative and advocate for patients within said team. The communication barriers intrinsic to the clinical hierarchy must be broken down, both within specialties and between professions [5]. Healthcare professionals must not only be encouraged to speak up for patient safety, but also feel individually accountable for the patient regardless of their role within the team. It is imperative to recognize that each team member provides value to patient care, whether it is knowing additional history that helps with approaching the patient or catching mistakes in the care plan. This philosophy lies at the root of effective IPT and is best ingrained early on in undergraduate medical training by incorporating longitudinal IPE.
By training together as students, future nurses, physicians, pharmacists, and social workers will inherently feel comfortable communicating with and correcting one another.

To accomplish this, we can glean insights from other industries. The healthcare industry has already begun to adopt principles from the automotive industry such as the lean principles of reducing waste and waiting times [6]. For example, at Toyota, every assembly line worker is empowered to stop the entire line if he or she notices a defect. In implementing this policy, managers noticed a long-term increase in overall efficiency and productivity. In addition, managers saw a shift of culture away from simply making cars to improving the process of making cars. The andon, or stop the line, approach shifted the overall assessment of quality from an individual in charge (Chief Safety Officer) to all individuals on the manufacturing line. At Toyota, engineers fundamentally believe that their job is not simply to manufacture cars, but to learn to manufacture cars better. An example of applying this ideology to medical education is scheduling simulated patient encounters with groups of interdisciplinary students. Students would not only practice their roles as nurses or physicians but also step into each other's roles as pharmacists, physician's assistants, or social workers. This allows students to learn team dynamics and experience providing different aspects of patient care. This deeper understanding of various healthcare roles will garner deeper appreciation and respect for each team member. As a result, students will not only feel empowered to speak up, but also feel more comfortable getting feedback from or being corrected by other members of their team who speak up. Longitudinal incorporation of IPE would entrench this sense of empowerment in all healthcare professional students and offer a way to concretely apply the principles of the Toyota model in healthcare. Additionally, health professional schools must emphasize that students are not simply learning how to provide great health care, but also how to improve the process of providing care. Continuous improvement is most effective when a team of providers with different perspectives can work collaboratively to produce innovative solutions.

\section{Conclusion}

Successful practice of IPT engenders collective accountability for patient care. However, this can only be accomplished by developing longitudinal, integrated models for IPE that engage students of all health professional schools. Evolving students' view of IPT from an optional adjunct to a core tenant of their education and future practice creates a naturally collaborative environment and an inherent sense of empowerment to step in and act when concerned about others' work. Making IPE a habit of practice that prioritizes team-based care goes beyond adapting education to fit the needs of healthcare. Currently, it is difficult to assess the extent in which IPE has 
been implemented at medical schools across the country. Further research opportunities include developing a climate survey to better understand what IPE programs exist and measure changes in practice for students who went through these programs. By developing tools to implement and measure the impact of various IPE interventions, such as interprofessional patient simulations and interdisciplinary rounds, future medical curricula changes will not simply be adapted to fit the future needs of healthcare, but rather be focused on developing the adaptive system needed to cope with problems we cannot possibly yet imagine.

Acknowledgements We would like to thank Dr. Paula Ross at the University of Michigan Medical School for her guidance and patience during the development of this piece.

\section{Compliance with Ethical Standards}

Conflict of Interest On behalf of all authors, the corresponding author states that there is no conflict of interest.

\section{References}

1. Abu-Rish E, Kim S, Choe L, et al. Current trends in interprofessional education of health sciences students: a literature review. Journal of Interprofessional Care. 2012;26(6):444-51. https://doi.org/10.3109/ 13561820.2012 .715604$.

2. Bridges DR, Davidson RA, Odegard PS, Maki IV, Tomkowiak J. Interprofessional collaboration: three best practice models of interprofessional education. Medical Education Online. 2011;16:10. https://doi.org/10.3402/meo.v16i0.6035.

3. Margalit R, Keating-Lefler R, Collier D, et al. Advancing interprofessional education: a qualitative analysis of student and faculty reflections. Medical Science Educator. 2013;23(Suppl 3):462-71. https://doi.org/10.1007/BF03341669.

4. Bridges DR, Davidson RA, Odegard PS, Maki IV, Tomkowiak J. Interprofessional collaboration: three best practice models of interprofessional education. Med Educ Online. 2011 Apr;8:16.

5. Lerner BH. In a hospital hierarchy, speaking up is hard to do. The New York Times, April. 2007;17 http://www.nytimes.com/2007/04/ 17/health/17essa.html

6. Kim CS, et al. Lean health care: what can hospitals learn from a world-class automaker? J of Hosp Medcine May/June. 2006;1:3 